To be published in the peer reviewed journal Animation: Practice, Production and Process in 2011

\title{
Emptying Frames
}

This short essay brings together some thoughts about two films, both of which take as their starting point the photographic still image and use film to expand and question the immobility of that image, teasing out small shifts and changes in its appearance. What follows are some observations about how these films reflect on their photographic materiality, the relationship between the still and moving image, the filmic interval, and the film and its projector. In the context of animation both films explore the boundaries of what constitutes the form. What are the most minimal conditions for 'animation' to take place? Can movement come from a single still image? In Candle (2007) and Tidal (2007), two obsolete and disappearing media come together to reflect on their own condition, their material and temporality.

Candle is a film that documents a Polaroid developing. It is shot on a $100 \mathrm{ft}$ roll of black and white $16 \mathrm{~mm}$ film. This standard film length is roughly equal to the time it takes for the Polaroid to fully develop, about 3 minutes. The film presents this photographic event in reverse. Over the duration of the film the image of a candle disappears back into the emulsion of the photograph. The initial intention for this film was that I wanted to capture all the images that emerge from the photographs development; the trace of the image as it solidifies into a fixed moment. The developing image is a time-based event and acts as an interval between the taking of the picture and the final settled image. Film preserves this moment, and makes possible it's manipulation.

There is an uncertainty about the temporality of the image. The developing process is not entirely clear to the viewer, the familiar white border of the Polaroid is not included in the film frame, and so it's difficult to work out what is taking place. It looks a little like a collision of two film transitions, a dissolve and fade combined. Candle attempts to create a dialogue between the filmic interval and the Polaroid's photographic interval. I wanted to put the two media together to see how they would react with each other and to see if the Polaroid's chemical change can materialise and articulate the filmic interval.

A key consideration with this film was the question of what kind of image to use, and how a dialogue could be created between it and the process. Initially I was looking for things that would reflect the idea of 'emergence' or 'becoming' that the photographic transformation symbolises, the images had to refer to a transition or change of some kind. To begin with I photographed a rainbow, then a number of trees in blossom, but the effect was unsatisfactory. The image needed to be able to suggest movement; it had to be an essential characteristic of the object being photographed. I finally settled on making a photograph of a candle, its flicker being the source of its animation. The idea of the melting of the candle merges with the photographic change of the Polaroid creating a paradox where the screen lightens as the candle 'melts' away till the flame is no more. 
Movement can be seen and imagined in the film. Firstly, there is a movement in the image that comes from the motion of the film stock as it records. Both of these films are shot on a Canon Scoopic that has a fairly unstable means of holding the film as each frame is being exposed. This accounts for a bit of a wobble in the image that becomes particularly pronounced when you film a still object/image at $24 \mathrm{fps}$. There is also a little more residual movement that comes from the projecting of the film, again for mechanical reasons. Finally, through conditioning the viewer expects to see movement on the screen, when confronted with a static image there is a desire to see it move, an element of 'projection' comes from the viewer.

The film projector is an important component in the screening of Candle as it sets up a discourse between the machine and the film. I'm interested in creating a dialogue between these two light sources, the light of the projector, and the 'light' of the candle. The projector is projecting yet also appears to be burning away the image of the candle. The projector illuminates the room more and more as the candle disappears.

Tidal also shot on a $100 \mathrm{ft}$ roll of $16 \mathrm{~mm}$ film explores the same ideas about materiality and duration as Candle but as a split screen film. A wave gradually disappears from one frame to the other over three minutes. The making of this film required some modification to the camera. A small piece of black $8 \mathrm{~mm}$ leader is placed vertically over the camera gate to create a mask; this allows one side of the film to be exposed then the other side. This process is the same principal as shooting standard $8 \mathrm{~mm}$ film where a $25 \mathrm{ft}$ roll of $16 \mathrm{~mm}$ is exposed on both sides and split down the middle to create $50 \mathrm{ft}$ of $8 \mathrm{~mm}$ film.

This technique is also similar to the pre-digital trick shot of masking the frame to create the illusion that the same person appears multiple times in a space, for example in Maya Deren's Meshes of the Afternoon (1943). We also see this kind of special effect in Georges Mélèis work at the dawn of the cinema. Yet the masking in Tidal is overtly visual, there is not attempt to hide the 'trick', the wave appears to spill over and underneath it.

In Candle the emphasis is on the changing light and it's relationship to the subject. In Tidal the manipulation of space is more prominent. The wave shifts from one frame into another, vey slowly. There is a moment in the middle of the film where both waves are at an equal stage of addition and subtraction. The film becomes suspended for a moment as we reach its central point before the wave continues its drift along into the right side of the frame. The wave appears to wash over the surface of the photograph/film as the chemistry brings forth the image. This chemical process and its relationship to tides and the sea are made explicit by the films title. 
The perceived movement in Tidal as with Candle comes from what the subject evokes in the mind of the viewer, the chemical transformation, the camera and projector faults, but also the shift in the screen from left to right, the viewer shifts their gaze across the screen. Movement is more prominent because we have something to measure it against, we can compare the two waves against each other to gauge where we are in the film.

The classic notion of animation brings drawings and objects to life by a series of small shifts or changes to them; the result is smooth and continuous movement. These shifts become 'invisible' and the space between each frame is lost or covered up by the illusion of movement. Tidal and Candle both attempt to slow down that shift, to get inside it, to be between the frames.

In Candle the photographic transformation is an attempt to articulate and materialize the space between the film frames. Tidal makes this more explicit by presenting this 'space' on screen as a vertical divide between the waves. The chemical change of the image in both Candle and Tidal creates a metaphor for movement and loss, evoking the alchemy of the photographic as it becomes a memory in a digital age. These two films present the beginning and end of an image, and both engage with the fundamental paradox at the core of cinema; that movement comes/came out of a series of still images.

Neil Henderson 